\title{
EFEITO DO NÍVEL DE INGESTÃO SOBRE A DIGESTIBILIDADE DOS AMINOÁCIDOS EM FRANGOS DE CORTE
}

\author{
Effect of the food intake on the amino acids digestibility in broiler chickens
}

\author{
Flávia Maria de Oliveira Borges ${ }^{1}$, Horácio Santiago Rostagno², \\ Carlos Eduardo do Prado Saad ${ }^{3}$, Leonardo Boscolli Lara ${ }^{4}$, Edgar Aleixo Teixeira ${ }^{5}$
}

\begin{abstract}
RESUMO
Foi realizado um trabalho com aves cecectomizadas para avaliar o efeito do nível de consumo sobre os coeficientes de digestibilidade dos aminoácidos (coeficiente de digestibilidade aparente (CDAaa) e coeficiente de digestibilidade verdadeira (CDVaa)), utilizando-se a metodologia de alimentação forçada. O delineamento experimental foi inteiramente ao acaso, em esquema fatorial 2 x 8 (níveis de consumo x alimentos), com quatro repetições. Sete alimentos oriundos do trigo e uma raçãoreferência foram utilizados. Todas as aves foram submetidas a jejum de 30 horas e forçadas a ingerir 25 e 50 gramas dos alimentos puros de uma única vez. Quatro aves em cada nível de consumo foram deixadas em jejum total para a determinação das perdas metabólica e endógena. Nos alimentos e excretas foram analisadas matéria seca, proteína bruta e aminoácidos. As médias foram comparadas pelo teste de SNK. Os valores de CDAaa para todos tratamentos foram afetados pela quantidade de alimento ingerido, e no nível de consumo menor os valores de CDAaa foram significativamente menores $(\mathrm{P}<0,05)$. Igualmente, a CDVaa apresentou diferenças significativas $(\mathrm{P}<0,05)$ entre alguns tratamentos, indicando que os níveis de consumo podem afetar esta medida.
\end{abstract}

TERMOS PARA INDEXAÇÃO: Aves, alimentação forçada, níveis de consumo, aminoácidos digestíveis, cecectomia.

\begin{abstract}
This paper were conducted with the objective of evaluation the effects of feed intake levels on the methodology of forced feeding of caecectomised broilers, on the coefficients of digestibility amino acids (apparent digestibility amino acids coefficients - ADCaa and true digestibility amino acids coefficients - TDCaa), using as feeds tests the wheat and some of its products. The feeds were randomly distributed in factorial outline of 2 x 8, (levels $\mathrm{x}$ feeds) with four repetitions. Seven wheat grain by-products were used. All broilers were submitted to a fast of 30 hours and forced to ingest 25 and 50 grams of the pure feeds ate the same time. In the two levels a treatment was left in fast, for the determination of the metabolic and endogenous losses. In the feeds and excrete, dry matter, crude protein and amino acids were analyzed. The means were compared by the test of SNK test. The values of DACaa over all treatments were affected for the amount of ingested feed, and in the lower intake level the values of DACaa were significantly lower $(\mathrm{P}, 0,05)$. In the same way, TDCaa showed significant differences $(\mathrm{P}<0,05)$ among some treatments, indicating that the feed intake levels should be take in account since it had effect on the digestibility of AA of caecectomised broilers.
\end{abstract}

INDEX TERMS: broilers , forced feeding, feed intake, digestible amino acids, caecectomy.

(Recebido para publicação em 6 de abril de 2004 e aprovado 10 de março de 2005)

\section{INTRODUÇÃO}

Da mesma maneira que o consumo pode afetar a energia metabolizável verdadeira (BORGES et al., 1990), avaliada pela metodologia de alimentação forçada (SIBBALD, 1976), ele poderia influenciar a avaliação de aminoácidos digestíveis, uma vez que a ingestão de pequena quantidade de alimento poderia induzir um catabolismo corporal, no sentido de suprir as necessidades energéticas. Esse catabolismo levaria a um aumento da porção de aminoácidos endógenos das aves, com uma subestimativa dos aminoácidos digestíveis dos alimentos.

Outra questão é a avaliação da fração endógena de nitrogênio. O método direto, baseado na alimentação de grupos de animais com dietas livres de proteína, ou mesmo em jejum, é a forma mais simples para estimar o nitrogênio endógeno.

\footnotetext{
1. Medica Veterinária - M.Sc.,Dra em Nutrição Animal - Universidade Federal de Lavras/UFLA - Caixa Postal 3037 - 37200000 - Lavras, MG borgesvet@ufla.br

2. Engenheiro Agrônomo - M.Sc., PhD Nutrição Animal - Departamento de Zootecnia - Universidade Federal de Viçosa/UFV - $36.570-000$ - Viçosa, MG.

3. Zootecnista - MSc., Dr Nutrição Animal - Fundação Zoobotânica de Belo Horizonte.

4. Médico Veterinário - Estudante de Doutorado da Universidade Federal de Lavras/UFLA.

5. Médico Veterinário - Estudante de Doutorado da Universidade Federal de Minas Gerais/UFMG
} 
Entretanto, esse método tem sido questionado porque o processo digestivo apresenta um comportamento diferente daquele que teria com o fornecimento de uma dieta normal; as excreções encontram-se diminuídas com tais rações, o que resultaria numa avaliação subestimada das perdas dos aminoácidos endógenos totais.

Vários autores questionam a grande variabilidade dos dados obtidos em aves em jejum, na qual a consideração da média desses valores poderia induzir a um erro não quantificável no modelo. As aves alimentadas com diferentes quantidades de alimentos poderiam apresentar excreções endógenas distintas entre si, bem como distintas daquelas em regime de restrição total de alimentos. Essas últimas apresentam um grande desvio padrão nos valores de excreção endógena, o que certamente poderia levar a uma sub ou superestimativa dos valores de aminoácidos digestíveis (DALE \& FULLER, 1984; PESTI et al., 1988). Sibbald (1980) discorda citando que aves submetidas ao jejum primeiro utilizam suas reservas lipídicas como fonte de energia e depois os tecidos protéicos. Desse modo, é essencial que a duração do período de jejum seja idêntico para o grupocontrole e o grupo-experimental.

Com trabalho teve-se como objetivo avaliar o efeito da quantidade de alimento ingerida sobre a digestibilidade aparente e verdadeira de aminoácidos de alimentos para frangos de corte adultos cecectomizados, utilizando-se metodologia de alimentação forçada.

\section{MATERIAL E MÉTODOS}

Este experimento foi conduzido nas dependências do departamento de Zootecnia da Escola de Veterinária da Universidade Federal de Minas Gerais (UFMG), Campus da Pampulha, em Belo Horizonte, com o objetivo de comparar o efeito de dois níveis de consumo de alimento (25 e 50 gramas) sobre os coeficientes de digestibilidade obtidos pela metodologia de alimentação forçada (SIBBALD, 1976).

Para tal experimento, foram utilizadas 32 aves previamente cecectomizadas, segundo Pupa et al. (1998), recebendo oito dietas, em dois períodos experimentais. Além das 32 aves, oito aves (quatro aves em cada período) foram utilizadas para estimar as perdas endógenas e metabólicas, e este grupo não fez parte das análises estatísticas.

Foram comparados dois níveis de consumo (25 e 50 gramas) utilizando-se uma ração-referência e sete alimentos-teste, constituídos de produtos oriundos do trigo: trigo integral moído (TIM), gérmen de trigo (GT), farinha de trigo clara (FTC), farinha de trigo escura (FTE), farelo de trigo para uso humano (FTH), farelo de trigo para uso animal (FTA) e farelo de trigo grosso (FTG),utilizando-se o método da alimentação forçada descrito por Sibbald (1976). A dieta-referência constituiu um tratamento e foi calculada segundo recomendações de Rostagno et al. (1985) a base de milho moído, farelo de soja, óleo de soja e mistura vitamínicomineral.

Os dois períodos experimentais foram de quatro dias cada um, obedecendo ao seguinte cronograma: todas as aves tiveram toda a região da cloaca previamente depenada para evitar aderência da excreta; .foram submetidas ao jejum de 30 horas para esvaziamento completo do trato gastrintestinal e após o jejum foram forçadas a ingerir 25 e 50 gramas dos alimentos teste de uma única vez. Foram utilizados os alimentos puros, sem fração de substituição, conforme recomendado por Sibbald (1976). Quatro aves foram deixadas em jejum por mais 48 horas para determinação das perdas metabólicas e endógenas.

A coleta de excreta foi total, de 12 em 12 horas, até completar o período exato de 48 horas para todas as aves, inclusive as em jejum. No final do experimento foram agrupadas por repetição e homogeneizadas para futuras análises.

As análises de matéria seca e aminoácidos foram realizadas no laboratório de nutrição animal da Escola de Veterinária da UFMG para os alimentos puros, ração-referência e excreta. As análises de aminoácidos foram feitas por hidrólise ácida e leitura por cromatografia líquida de alta eficiência (CLAE) em coluna de troca iônica com reação pós-coluna com ortoftaloleido (OPA) nos alimentos puros, ração-referência e excreta, utilizando-se cromatógrafo de fase líquida Shimatzu - 10A, com detector de fluorescência (MOTTER, 1991).

Nos alimentos puros foram analisados os aminoácidos essenciais: valina (VAL), metionina (MET), isoleucina (ISO), leucina (LEU), treonina (THR), fenilalanina (FEN), histidina (HIST), lisina (LYS) e arginina (ARG), e os não essenciais aspartato (ASP), tirosina (TYR), serina (SER), glutamina (GLU), prolina (PRO), glicina (GLI), alanina (ALA) ecistina (CYS) conforme a Tabela 1.

As fórmulas utilizadas para os cálculos dos coeficientes de aminoácidos digestíveis aparentes e verdadeiros dos alimentos foram às citadas por Albino (1991). 
BORGES, F. M. de O. et al.

TABELA 1 - Composição em aminoácidos essenciais e não essenciais (\%/MS) dos alimentos.

\begin{tabular}{lcccccccc}
\hline \multicolumn{1}{c}{ Aminoácido } & RR & TIM & GT & FTC & FTE & FTH & FTA & FTG \\
\hline Valina & 0,99 & 0,66 & 0,86 & 0,43 & 0,36 & 0,75 & 0,55 & 0,67 \\
Metionina & 0,48 & 0,23 & 0,36 & 0,14 & 0,15 & 0,24 & 0,17 & 0,21 \\
Isoleucina & 0,89 & 0,52 & 1,00 & 0,76 & 0,24 & 1,07 & 0,40 & 1,02 \\
Leucina & 2,04 & 1,16 & 1,77 & 0,80 & 0,85 & 1,10 & 0,86 & 1,07 \\
Treonina & 0,78 & 0,39 & 0,74 & 0,20 & 0,31 & 0,44 & 0,34 & 0,44 \\
Fenilalanina & 1,31 & 0,93 & 1,26 & 0,63 & 0,65 & 0,81 & 0,68 & 0,80 \\
Histidina & 0,87 & 0,56 & 1,21 & 0,34 & 0,41 & 0,65 & 0,52 & 0,59 \\
Lisina & 1,62 & 0,56 & 2,32 & 0,32 & 0,45 & 0,99 & 0,74 & 0,72 \\
Arginina & 2,35 & 1,06 & 2,99 & 0,53 & 0,76 & 1,85 & 1,24 & 1,51 \\
Aspartato & 2,55 & 0,81 & 2,94 & 0,47 & 0,7 & 1,26 & 0,96 & 1,02 \\
Tirosina & 0,94 & 0,52 & 1,13 & 0,33 & 0,37 & 0,63 & 0,49 & 0,54 \\
Serina & 1,26 & 0,86 & 1,46 & 0,59 & 0,71 & 0,81 & 0,64 & 0,81 \\
Glutamina & 5,11 & 7,05 & 5,44 & 5,33 & 5,3 & 3,86 & 3,28 & 5,19 \\
Prolina O & 1,64 & 2,25 & 1,63 & 1,69 & 1,53 & 1,26 & 1,03 & 1,71 \\
Glicina I & 0,91 & 0,64 & 0,71 & 0,4 & 0,25 & 0,87 & 0,63 & 0,78 \\
Alanina & 1,07 & 0,52 & 1,92 & 0,31 & 0,51 & 0,82 & 0,56 & 0,64 \\
Cistina & 0,25 & 0,25 & 0,23 & 0,14 & 0,19 & 0,2 & 0,15 & 0,23 \\
\hline
\end{tabular}

Análises realizadas no laboratório de nutrição da Escola de Veterinária da UFMG

1: RR = ração-referência, TIM = trigo integral moído, GT = germe de trigo, FTC = farinha de trigo clara, FTE = farinha de trigo escura, FTH = farelo de trigo para uso humano, FTA = farelo de trigo para uso animal, FTG = farelo de trigo grosso.

Para a avaliação dos efeitos do nível de consumo sobre a digestibilidade dos aminoácidos determinados pela metodologia de alimentação forçada, adotou-se um, delineamento experimental em blocos inteiramente casualizados, com oito tratamentos, sendo cada ave uma unidade experimental, durante dois períodos experimentais, em esquema fatorial $2 \times 8$ (níveis de consumo $\mathrm{x}$ alimentos), com quatro repetições por tratamento, totalizando 64 repetições. As médias foram comparadas pelo teste de $\mathrm{F}$.

\section{RESULTADOS E DISCUSSÃO}

Nas Tabelas 2 e 3 encontram-se descritos os valores de digestibilidade aparente dos aminoácidos essenciais (CDAaae) e não essenciais (CDAaane) em porcentagem, respectivamente, segundo o nível de consumo. O maior nível de ingestão (50 gramas) apresentou maiores valores de CDAaa ${ }_{\mathrm{e}}$ e de CDAaa ${ }_{\text {ne }}$ para todos os aminoácidos $(\mathrm{P}<0,05)$, com exceção da isoleucina da ração-referência. Embora não tenham sido encontrados, na literatura disponível, trabalhos com relação ao efeito do consumo sobre a CDAaa, pode-se presumir que, igual à energia metabolizável (BORGES et al., 1990), os valores de CDAaa estão sujeitos ao efeito do nível de consumo. A influência dos aminoácidos metabólicos e endógenos (Tabela 5) é mais alta em baixo nível de consumo, o que leva a uma subestimativa da digestibilidade aparente dos aminoácidos dos alimentos no nível de consumo menor. Essa influência diminui com o aumento 
da ingestão, conforme pode ser observado neste experimento. Entretanto, segundo Sibbald (1977), na metodologia de alimentação forçada, desafortunadamente não se pode ultrapassar as 50 gramas de alimento, sob o risco de provocar regurgitação e contaminar a excreta .

Os valores negativos de digestibilidade aparente de alguns aminoácidos essenciais (isoleucina e treonina na FTE e treonina na FTC) encontrados no tratamento com 25 gramas de alimento reforçam o efeito do consumo. Os valores desses aminoácidos nos alimentos são muito baixos, comparados aos demais $(0,20$ e 0,31\% de treonina na FTC e FTE, respectivamente, e $0,24 \%$ de isoleucina na FTE). Isso faz com que a excreção endógena (tabela 8) subestime a digestibilidade desses aminoácidos.

Os alimentos fibrosos apresentam uma tendência, não avaliada estatisticamente, de apresentar menores valores de CDAaa nos dois níveis de ingestão, particularmente no nível de mais baixo consumo, devido à interferência da porção endógena, aliada à baixa digestibilidade desses alimentos. De acordo com Mejía \& Ferreira (1996), o teor e o tipo de fibra promovem aumento das secreções endógenas, o que pode contribuir para uma maior excreção endógena em aves alimentadas com alta fibra, o que levaria a uma subestimativa da digestibilidade dos aminoácidos.

Segundo Mejía \& Ferreira (1996), mesmo que a metodologia de utilização de aves em jejum ou alimentadas com dietas purificadas livres de proteína seja a forma mais simples e fácil para estimar o nitrogênio endógeno, esse método tem sido muito questionado porque o processo digestivo apresenta um comportamento diferente daquele que teria com o fornecimento de uma dieta normal. Os autores citam que as excreções encontram-se diminuídas com tais rações, o que resultaria numa subavaliação das perdas dos aminoácidos endógenos totais.

TABELA 2 - Coeficiente de digestibilidade aparente (\%) dos aminoácidos essenciais dos alimentos ${ }^{1}$, segundo o nível de consumo.

\begin{tabular}{lccccccccc}
\hline \multirow{2}{*}{ Aminoácido } & Método & RR & TIM & GT & FTC & FTE & FTH & FTA & FTG \\
\hline \multirow{2}{*}{ Valina } & Trat. 25g & $65,24 \mathrm{~b}$ & $48,93 \mathrm{~b}$ & $53,43 \mathrm{~b}$ & $42,19 \mathrm{~b}$ & $29,08 \mathrm{~b}$ & $51,71 \mathrm{~b}$ & $42,48 \mathrm{~b}$ & $45,92 \mathrm{~b}$ \\
& Trat. 50g & $80,73 \mathrm{a}$ & $75,26 \mathrm{a}$ & $78,13 \mathrm{a}$ & $74,04 \mathrm{a}$ & $53,17 \mathrm{a}$ & $67,48 \mathrm{a}$ & $68,33 \mathrm{a}$ & $63,06 \mathrm{a}$ \\
\hline \multirow{2}{*}{ Metionina } & Trat. 25g & $82,96 \mathrm{~b}$ & $61,60 \mathrm{~b}$ & $69,53 \mathrm{~b}$ & $36,89 \mathrm{~b}$ & $55,53 \mathrm{~b}$ & $56,73 \mathrm{~b}$ & $44,95 \mathrm{~b}$ & $59,74 \mathrm{~b}$ \\
& Trat. 50g & $94,40 \mathrm{a}$ & $87,98 \mathrm{a}$ & $90,75 \mathrm{a}$ & $82,96 \mathrm{a}$ & $71,43 \mathrm{a}$ & $87,73 \mathrm{a}$ & $89,34 \mathrm{a}$ & $89,52 \mathrm{a}$ \\
\hline \multirow{2}{*}{ Isoleucina } & Trat. 25g & $61,97 \mathrm{a}$ & $40,38 \mathrm{~b}$ & $70,37 \mathrm{~b}$ & $67,25 \mathrm{~b}$ & $-18,46 \mathrm{~b}$ & $64,22 \mathrm{~b}$ & $21,95 \mathrm{~b}$ & $65,51 \mathrm{~b}$ \\
& Trat. 50g & $68,32 \mathrm{a}$ & $57,52 \mathrm{a}$ & $82,61 \mathrm{a}$ & $79,75 \mathrm{a}$ & $35,51 \mathrm{a}$ & $71,69 \mathrm{a}$ & $38,86 \mathrm{a}$ & $71,59 \mathrm{a}$ \\
\hline \multirow{2}{*}{ Leucina } & Trat. 25g & $61,38 \mathrm{~b}$ & $37,51 \mathrm{~b}$ & $59,28 \mathrm{~b}$ & $20,77 \mathrm{~b}$ & $8,95 \mathrm{~b}$ & $6,80 \mathrm{~b}$ & $9,74 \mathrm{~b}$ & $9,06 \mathrm{~b}$ \\
& Trat. 50g & $92,06 \mathrm{a}$ & $89,21 \mathrm{a}$ & $93,04 \mathrm{a}$ & $89,98 \mathrm{a}$ & $85,67 \mathrm{a}$ & $84,49 \mathrm{a}$ & $83,37 \mathrm{a}$ & $84,05 \mathrm{a}$ \\
\hline \multirow{2}{*}{ Treonina } & Trat. 25g & $52,09 \mathrm{~b}$ & $5,65 \mathrm{~b}$ & $50,91 \mathrm{~b}$ & $-70,58 \mathrm{~b}$ & $-13,48 \mathrm{~b}$ & $3,185 \mathrm{~b}$ & $0,465 \mathrm{~b}$ & $12,52 \mathrm{~b}$ \\
& Trat. 50g & $85,65 \mathrm{a}$ & $76,18 \mathrm{a}$ & $88,68 \mathrm{a}$ & $70,98 \mathrm{a}$ & $70,58 \mathrm{a}$ & $76,58 \mathrm{a}$ & $75,15 \mathrm{a}$ & $77,55 \mathrm{a}$ \\
\hline \multirow{2}{*}{ Fenilalanina } & Trat. 25g & $55,77 \mathrm{~b}$ & $40,29 \mathrm{~b}$ & $53,74 \mathrm{~b}$ & $30,42 \mathrm{~b}$ & $23,17 \mathrm{~b}$ & $29,79 \mathrm{~b}$ & $22,37 \mathrm{~b}$ & $35,71 \mathrm{~b}$ \\
& Trat. 50g & $82,62 \mathrm{a}$ & $80,26 \mathrm{a}$ & $81,76 \mathrm{a}$ & $78,17 \mathrm{a}$ & $72,83 \mathrm{a}$ & $70,79 \mathrm{a}$ & $71,82 \mathrm{a}$ & $69,26 \mathrm{a}$ \\
\hline \multirow{2}{*}{ Histidina } & Trat. 25g & $52,88 \mathrm{~b}$ & $35,81 \mathrm{~b}$ & $75,31 \mathrm{~b}$ & $8,24 \mathrm{~b}$ & $27,88 \mathrm{~b}$ & $14,02 \mathrm{~b}$ & $26,55 \mathrm{~b}$ & $29,02 \mathrm{~b}$ \\
& Trat. 50g & $84,35 \mathrm{a}$ & $80,13 \mathrm{a}$ & $86,54 \mathrm{a}$ & $71,60 \mathrm{a}$ & $71,30 \mathrm{a}$ & $68,84 \mathrm{a}$ & $75,09 \mathrm{a}$ & $69,75 \mathrm{a}$ \\
\hline \multirow{2}{*}{ Lisina } & Trat. 25g & $79,27 \mathrm{~b}$ & $41,54 \mathrm{~b}$ & $85,77 \mathrm{~b}$ & $11,04 \mathrm{~b}$ & $43,85 \mathrm{~b}$ & $60,55 \mathrm{~b}$ & $47,08 \mathrm{~b}$ & $51,59 \mathrm{~b}$ \\
& Trat. 50g & $87,72 \mathrm{a}$ & $74,48 \mathrm{a}$ & $90,95 \mathrm{a}$ & $72,83 \mathrm{a}$ & $64,79 \mathrm{a}$ & $75,02 \mathrm{a}$ & $72,22 \mathrm{a}$ & $68,03 \mathrm{a}$ \\
\hline
\end{tabular}

Valores na coluna, para uma mesma variável, com letras distintas diferem entre si, pelo teste $\mathrm{F}(\mathrm{P}<0,05)$.

1: $\mathrm{RR}=$ ração-referência, TIM = trigo integral moído, GT = germe de trigo, FTC = farinha de trigo clara, FTE = farinha de trigo escura, FTH = farelo de trigo para uso humano, FTA = farelo de trigo para uso animal, FTG = farelo de trigo grosso. 
TABELA 3 - Coeficiente de digestibilidade aparente (\%) dos aminoácidos não essenciais dos alimentos ${ }^{1}$, segundo o nível de consumo.

\begin{tabular}{cccccccccc}
\hline \multirow{2}{*}{ Aminoácido } & Método & \multirow{2}{*}{ RR } & TIM & GT & FTC & FTE & FTH & FTA & FTG \\
\hline \multirow{2}{*}{ Aspartato } & Trat. 25g & $67,43 \mathrm{~b}$ & $16,25 \mathrm{~b}$ & $75,00 \mathrm{~b}$ & $-29,29 \mathrm{~b}$ & $15,99 \mathrm{~b}$ & $28,25 \mathrm{~b}$ & $25,72 \mathrm{~b}$ & $31,41 \mathrm{~b}$ \\
& Trat. 50g & $87,42 \mathrm{a}$ & $70,31 \mathrm{a}$ & $90,41 \mathrm{a}$ & $61,91 \mathrm{a}$ & $63,50 \mathrm{a}$ & $71,50 \mathrm{a}$ & $70,71 \mathrm{a}$ & $65,43 \mathrm{a}$ \\
\hline \multirow{2}{*}{ Tirosina } & Trat. 25g & $55,14 \mathrm{~b}$ & $19,16 \mathrm{~b}$ & $64,51 \mathrm{~b}$ & $0,55 \mathrm{~b}$ & $3,14 \mathrm{~b}$ & $24,85 \mathrm{~b}$ & $22,72 \mathrm{~b}$ & $21,65 \mathrm{~b}$ \\
& Trat. 50g & $79,33 \mathrm{a}$ & $69,50 \mathrm{a}$ & $84,22 \mathrm{a}$ & $58,39 \mathrm{a}$ & $46,66 \mathrm{a}$ & $64,36 \mathrm{a}$ & $63,08 \mathrm{a}$ & $62,13 \mathrm{a}$ \\
\hline \multirow{2}{*}{ Sererina } & Trat. 25g & $63,83 \mathrm{~b}$ & $46,19 \mathrm{~b}$ & $70,21 \mathrm{~b}$ & $37,50 \mathrm{~b}$ & $44,96 \mathrm{~b}$ & $37,74 \mathrm{~b}$ & $35,60 \mathrm{~b}$ & $42,37 \mathrm{~b}$ \\
& Trat. 50g & $84,37 \mathrm{a}$ & $78,85 \mathrm{a}$ & $87,21 \mathrm{a}$ & $76,94 \mathrm{a}$ & $72,38 \mathrm{a}$ & $71,98 \mathrm{a}$ & $70,70 \mathrm{a}$ & $73,48 \mathrm{a}$ \\
\hline \multirow{2}{*}{ Glutamina } & Trat. 25g & $78,19 \mathrm{~b}$ & $85,44 \mathrm{~b}$ & $83,48 \mathrm{~b}$ & $84,14 \mathrm{~b}$ & $84,65 \mathrm{~b}$ & $71,17 \mathrm{~b}$ & $69,78 \mathrm{~b}$ & $77,79 \mathrm{~b}$ \\
& Trat. 50g & $90,06 \mathrm{a}$ & $94,07 \mathrm{a}$ & $91,39 \mathrm{a}$ & $94,58 \mathrm{a}$ & $92,06 \mathrm{a}$ & $84,39 \mathrm{a}$ & $89,91 \mathrm{a}$ & $88,57 \mathrm{a}$ \\
\hline \multirow{2}{*}{ Prolina } & Trat. 25g & $63,99 \mathrm{~b}$ & $71,09 \mathrm{~b}$ & $65,34 \mathrm{~b}$ & $70,02 \mathrm{~b}$ & $62,11 \mathrm{~b}$ & $46,26 \mathrm{~b}$ & $47,10 \mathrm{~b}$ & $67,23 \mathrm{~b}$ \\
& Trat. 50g & $84,00 \mathrm{a}$ & $89,27 \mathrm{a}$ & $83,82 \mathrm{a}$ & $87,22 \mathrm{a}$ & $81,82 \mathrm{a}$ & $76,21 \mathrm{a}$ & $75,46 \mathrm{a}$ & $83,27 \mathrm{a}$ \\
\hline \multirow{2}{*}{ Alanina } & Trat. 25g & $60,03 \mathrm{~b}$ & $14,78 \mathrm{~b}$ & $77,38 \mathrm{~b}$ & $-2,39 \mathrm{~b}$ & $33,32 \mathrm{~b}$ & $36,82 \mathrm{~b}$ & $19,58 \mathrm{~b}$ & $15,28 \mathrm{~b}$ \\
& Trat. 50g & $79,76 \mathrm{a}$ & $66,24 \mathrm{a}$ & $86,59 \mathrm{a}$ & $60,98 \mathrm{a}$ & $65,58 \mathrm{a}$ & $66,62 \mathrm{a}$ & $60,15 \mathrm{a}$ & $58,33 \mathrm{a}$ \\
\hline
\end{tabular}

Valores na coluna, para uma mesma variável, com letras distintas diferem entre si, pelo teste $\mathrm{F}(\mathrm{P}<0,05)$

1: $\mathrm{RR}=$ ração-referência, TIM = trigo integral moído, GT = germe de trigo, FTC = farinha de trigo clara, FTE = farinha de trigo escura, FTH = farelo de trigo para uso humano, FTA = farelo de trigo para uso animal, FTG = farelo de trigo grosso.

O mesmo comportamento também é observado nos valores de digestibilidade aparente da soma total dos aminoácidos, da soma dos aminoácidos não essenciais, da soma dos aminoácidos essenciais, e da soma dos aminoácidos limitantes (Met + Thr + Lys), em porcentagem, segundo o nível de consumo (Tabela 4). As aves dos tratamentos com menor nível de consumo (25 gramas) apresentaram os menores valores de CDAaa em todos os alimentos $(\mathrm{P}<0,05)$ devido à alta interferência das perdas metabólicas e endógenas (Tabela 5) e do baixo consumo nos métodos de alimentação forçada.

Nas Tabelas 6 e 7 apresentam-se os valores de digestibilidade verdadeira dos aminoácidos essenciais $\left(C D V a a_{e}\right)$ e não essenciais $\left(C^{2} V_{a a}{ }_{n e}\right)$ em porcentagem, respectivamente, segundo o nível de consumo. O esperado, teoricamente, é que subtraído o efeito da porção endógena, as aves apresentassem a mesma digestibilidade verdadeira, uma vez que elas apresentavam características corporais e pesos vivos similares. Entretanto, não foi o que ocorreu, demonstrando que mesmo que se retire a porção endógena calculada pela média de excreção das aves em jejum, o nível de consumo continua a imprimir o seu efeito sobre a digestibilidade dos aminoácidos, embora esse efeito seja minimizado quando comparado ao efeito sobre a digestibilidade aparente.

A digestibilidade verdadeira para a maioria dos aminoácidos (tanto essenciais quanto não essenciais) foi maior no maior nível de consumo $(\mathrm{P}<0,05)$, com exceção da lisina, quando todos os alimentos apresentaram valores significativamente iguais $(\mathrm{P}>0,05)$ e somente a FTC apresentou maior valor de CDVaa para a lisina no maior nível de consumo. Esse comportamento pode ser observado com maior clareza nos valores de digestibilidade verdadeira da soma total dos aminoácidos, da soma dos aminoácidos não essenciais, da soma dos aminoácidos essenciais e da soma dos aminoácidos limitantes (Met + Thr + Lys), descritos na Tabela 8. 
TABELA 4 - Coeficiente de digestibilidade aparente (\%) da soma total dos aminoácidos (aa), da soma dos aa não essenciais, da soma dos aa essenciais e da soma dos aa limitantes dos alimentos ${ }^{1}$, segundo o nível de consumo.

\begin{tabular}{lccccccccc}
\hline \multirow{2}{*}{ Aminoácido } & Método & RR & TIM & GT & FTC & FTE & FTH & FTA & FTG \\
\hline \multirow{2}{*}{ Totais } & Trat. 25g & $64,23 \mathrm{~b}$ & $40,33 \mathrm{~b}$ & $69,98 \mathrm{~b}$ & $21,91 \mathrm{~b}$ & $28,72 \mathrm{~b}$ & $38,14 \mathrm{~b}$ & $32,41 \mathrm{~b}$ & $40,55 \mathrm{~b}$ \\
& Trat. 50g & $85,96 \mathrm{a}$ & $85,42 \mathrm{a}$ & $69,98 \mathrm{a}$ & $85,18 \mathrm{a}$ & $79,58 \mathrm{a}$ & $76,02 \mathrm{a}$ & $74,96 \mathrm{a}$ & $77,96 \mathrm{a}$ \\
\hline \multirow{2}{*}{$\mathrm{AA}_{\mathrm{NE}}{ }^{2}$} & Trat. 25g & $64,76 \mathrm{~b}$ & $42,16 \mathrm{~b}$ & $73,12 \mathrm{~b}$ & $26,75 \mathrm{~b}$ & $40,70 \mathrm{~b}$ & $41,15 \mathrm{~b}$ & $37,69 \mathrm{~b}$ & $42,62 \mathrm{~b}$ \\
& Trat. 50g & $87,19 \mathrm{a}$ & $89,20 \mathrm{a}$ & $89,06 \mathrm{a}$ & $88,60 \mathrm{a}$ & $84,32 \mathrm{a}$ & $78,09 \mathrm{a}$ & $78,18 \mathrm{a}$ & $82,18 \mathrm{a}$ \\
\hline \multirow{2}{*}{$\mathrm{AA}_{\mathrm{E}}{ }^{2}$} & Trat. 25g & $63,94 \mathrm{~b}$ & $38,96 \mathrm{~b}$ & $67,63 \mathrm{~b}$ & $18,28 \mathrm{~b}$ & $19,73 \mathrm{~b}$ & $35,88 \mathrm{~b}$ & $28,44 \mathrm{~b}$ & $39,00 \mathrm{~b}$ \\
& Trat. 50g & $84,55 \mathrm{a}$ & $77,55 \mathrm{a}$ & $87,22 \mathrm{a}$ & $77,87 \mathrm{a}$ & $69,24 \mathrm{a}$ & $73,65 \mathrm{a}$ & $70,69 \mathrm{a}$ & $71,60 \mathrm{a}$ \\
\hline \multirow{2}{*}{$\mathrm{LIM}^{2,3}$} & Trat. 25g & $71,44 \mathrm{~b}$ & $36,26 \mathrm{~b}$ & $72,39 \mathrm{~b}$ & $-7,55 \mathrm{~b}$ & $29,08 \mathrm{~b}$ & $40,16 \mathrm{~b}$ & $34,86 \mathrm{~b}$ & $42,26 \mathrm{~b}$ \\
& Trat. 50g & $88,28 \mathrm{a}$ & $77,80 \mathrm{a}$ & $90,43 \mathrm{a}$ & $74,43 \mathrm{a}$ & $67,87 \mathrm{a}$ & $77,22 \mathrm{a}$ & $75,36 \mathrm{a}$ & $74,38 \mathrm{a}$ \\
\hline
\end{tabular}

Valores na coluna, para uma mesma variável, com letras distintas diferem entre si, pelo teste $\mathrm{F}(\mathrm{P}<0,05)$

1: RR = ração-referência, TIM = trigo integral moído, GT = germe de trigo, FTC = farinha de trigo clara, FTE = farinha de trigo escura, FTH = farelo de trigo para uso humano, FTA = farelo de trigo para uso animal, FTG = farelo de trigo grosso.

2: $\mathrm{AA}_{\mathrm{NE}}=$ aminoácidos não essenciais, $\mathrm{AA}_{\mathrm{E}}=$ aminoácidos essenciais e $\mathrm{LIM}=$ aminoácidos limitantes

3: Metionina, treonina e lisina.

TABELA 5 - Valores de aminoácidos (mg) expressos por peso vivo total, por 100 gramas de peso vivo e em Kg de peso metabólico, do grupo de oito aves deixadas em jejum para a estimativa das perdas endógenas.

\begin{tabular}{cccc}
\hline Aminoácido & mg/aves em $\mathbf{2 4} \mathbf{h .}$ & $\mathbf{m g} / \mathbf{1 0 0 g} \mathbf{~ d e ~} \mathbf{P V}$ & $\mathbf{~ m g} / \mathbf{~ k g} \mathbf{P 0 , 7 5}$ \\
\hline Asp & 56,525 & 2,545 & 23,3 \\
Tyr & 32,085 & 1,375 & 12,965 \\
Ser & 36,13 & 1,53 & 14,535 \\
Glu & 76,97 & 3,205 & 30,945 \\
Pro & 50,7 & 2,11 & 20,37 \\
Ala & 33,22 & 1,345 & 13,35 \\
Val & 16,795 & 0,755 & 6,66 \\
Met & 6,535 & 0,285 & 2,63 \\
Iso & 23,3 & 1,02 & 9,385 \\
Leu & 65,155 & 2,86 & 26,24 \\
Thr & 22,62 & 1,19 & 10,59 \\
Fen & 39,135 & 1,725 & 15,88 \\
Hys & 24,145 & 1,08 & 9,85 \\
Lys & 24,865 & 1,115 & 10,15 \\
Totais & 512,505 & 22,16 & 206,34 \\
\hline
\end{tabular}


TABELA 6 - Coeficientes de digestibilidade verdadeira (\%) dos aminoácidos essenciais dos alimentos ${ }^{1}$, segundo o nível de consumo.

\begin{tabular}{lccccccccc}
\hline \multirow{2}{*}{ Aminoácido } & Método & RR & TIM & GT & FTC & FTE & FTH & FTA & FTG \\
\hline \multirow{2}{*}{ Valina } & Trat. 25g & $80,28 \mathrm{a}$ & $71,76 \mathrm{~b}$ & $82,73 \mathrm{a}$ & $77,76 \mathrm{~b}$ & $70,79 \mathrm{a}$ & $71,37 \mathrm{~b}$ & $69,14 \mathrm{a}$ & $68,44 \mathrm{a}$ \\
& Trat. 50g & $84,62 \mathrm{a}$ & $81,22 \mathrm{a}$ & $82,60 \mathrm{a}$ & $83,24 \mathrm{a}$ & $63,96 \mathrm{~b}$ & $72,56 \mathrm{a}$ & $75,23 \mathrm{a}$ & $68,88 \mathrm{a}$ \\
\hline \multirow{2}{*}{ Metionina } & Trat. 25g & $94,06 \mathrm{~b}$ & $85,69 \mathrm{~b}$ & $93,76 \mathrm{a}$ & $75,22 \mathrm{~b}$ & $92,28 \mathrm{~b}$ & $79,22 \mathrm{~b}$ & $82,43 \mathrm{~b}$ & $88,54 \mathrm{~b}$ \\
& Trat. 50g & $97,70 \mathrm{a}$ & $95,14 \mathrm{a}$ & $95,13 \mathrm{a}$ & $94,36 \mathrm{a}$ & $81,96 \mathrm{a}$ & $92,20 \mathrm{a}$ & $92,58 \mathrm{a}$ & $97,22 \mathrm{a}$ \\
\hline \multirow{2}{*}{ Isoleucina } & Trat. 25g & $83,51 \mathrm{a}$ & $78,50 \mathrm{a}$ & $89,56 \mathrm{a}$ & $92,96 \mathrm{~b}$ & $61,41 \mathrm{a}$ & $82,09 \mathrm{a}$ & $70,29 \mathrm{a}$ & $84,68 \mathrm{~b}$ \\
& Trat. 50g & $74,66 \mathrm{~b}$ & $68,75 \mathrm{~b}$ & $88,26 \mathrm{a}$ & $87,32 \mathrm{a}$ & $59,02 \mathrm{~b}$ & $76,09 \mathrm{~b}$ & $53,09 \mathrm{~b}$ & $77,23 \mathrm{a}$ \\
\hline \multirow{2}{*}{ Leucina } & Trat. 25g & $88,32 \mathrm{~b}$ & $85,84 \mathrm{~b}$ & $90,28 \mathrm{~b}$ & $90,48 \mathrm{a}$ & $74,38 \mathrm{~b}$ & $56,48 \mathrm{~b}$ & $72,87 \mathrm{~b}$ & $60,73 \mathrm{~b}$ \\
& Trat. 50g & $97,71 \mathrm{a}$ & $99,36 \mathrm{a}$ & $99,55 \mathrm{a}$ & $101,62 \mathrm{a}$ & $97,41 \mathrm{a}$ & $94,93 \mathrm{a}$ & $96,63 \mathrm{a}$ & $94,90 \mathrm{a}$ \\
\hline \multirow{2}{*}{ Treonina } & Trat. 25g & $85,74 \mathrm{~b}$ & $75,11 \mathrm{~b}$ & $86,45 \mathrm{~b}$ & $64,16 \mathrm{~b}$ & $70,51 \mathrm{~b}$ & $62,89 \mathrm{~b}$ & $77,82 \mathrm{~b}$ & $73,03 \mathrm{a}$ \\
& Trat. 50g & $92,40 \mathrm{a}$ & $90,13 \mathrm{a}$ & $95,82 \mathrm{a}$ & $98,04 \mathrm{a}$ & $87,45 \mathrm{a}$ & $88,58 \mathrm{a}$ & $90,69 \mathrm{a}$ & $79,70 \mathrm{a}$ \\
\hline \multirow{2}{*}{ Fenilalanina } & Trat. 25g & $80,59 \mathrm{~b}$ & $76,20 \mathrm{~b}$ & $79,59 \mathrm{~b}$ & $82,64 \mathrm{~b}$ & $73,71 \mathrm{~b}$ & $69,48 \mathrm{a}$ & $70,01 \mathrm{~b}$ & $77,01 \mathrm{~b}$ \\
& Trat. 50g & $89,96 \mathrm{a}$ & $90,88 \mathrm{a}$ & $89,41 \mathrm{a}$ & $93,62 \mathrm{a}$ & $87,79 \mathrm{a}$ & $82,68 \mathrm{a}$ & $85,91 \mathrm{a}$ & $81,49 \mathrm{a}$ \\
\hline \multirow{2}{*}{ Histidina } & Trat. 25g & $76,65 \mathrm{~b}$ & $73,77 \mathrm{~b}$ & $92,36 \mathrm{a}$ & $69,52 \mathrm{~b}$ & $68,26 \mathrm{~b}$ & $45,86 \mathrm{~b}$ & $66,13 \mathrm{~b}$ & $64,67 \mathrm{a}$ \\
& Trat. 50g & $91,06 \mathrm{a}$ & $90,84 \mathrm{a}$ & $91,38 \mathrm{a}$ & $88,87 \mathrm{a}$ & $85,50 \mathrm{a}$ & $77,81 \mathrm{a}$ & $86,25 \mathrm{a}$ & $69,80 \mathrm{a}$ \\
\hline \multirow{2}{*}{ Lisina } & Trat. 25g & $92,75 \mathrm{a}$ & $81,28 \mathrm{a}$ & $96,36 \mathrm{a}$ & $80,05 \mathrm{~b}$ & $92,48 \mathrm{a}$ & $82,48 \mathrm{a}$ & $81,95 \mathrm{a}$ & $82,27 \mathrm{a}$ \\
& Trat. 50g & $92,91 \mathrm{a}$ & $90,03 \mathrm{a}$ & $94,56 \mathrm{a}$ & $99,38 \mathrm{a}$ & $93,50 \mathrm{a}$ & $83,46 \mathrm{a}$ & $83,48 \mathrm{a}$ & $79,84 \mathrm{a}$ \\
\hline
\end{tabular}

Valores na coluna, para uma mesma variável, com letras distintas diferem entre si, pelo teste $\mathrm{F}(\mathrm{P}<0,05)$

1: RR = ração-referência, TIM = trigo integral moído, GT = germe de trigo, FTC = farinha de trigo clara, FTE = farinha de trigo escura, FTH = farelo de trigo para uso humano, FTA = farelo de trigo para uso animal, FTG = farelo de trigo grosso.

TABELA 7 - Coeficientes de digestibilidade verdadeira (\%) dos aminoácidos não essenciais dos alimentos ${ }^{1}$, segundo o nível de consumo.

\begin{tabular}{lccccccccc}
\hline \multirow{2}{*}{ Aminoácido } & Método & RR & TIM & GT & FTC & FTE & FTH & FTA & FTG \\
\hline \multirow{2}{*}{ Aspartato } & Trat. 25g & $87,40 \mathrm{~b}$ & $80,90 \mathrm{a}$ & $92,31 \mathrm{a}$ & $81,73 \mathrm{~b}$ & $89,45 \mathrm{~b}$ & $68,35 \mathrm{~b}$ & $78,35 \mathrm{~b}$ & $81,64 \mathrm{~b}$ \\
& Trat. 50g & $92,76 \mathrm{a}$ & $86,44 \mathrm{a}$ & $94,73 \mathrm{a}$ & $89,60 \mathrm{a}$ & $81,83 \mathrm{a}$ & $81,50 \mathrm{a}$ & $83,84 \mathrm{a}$ & $78,00 \mathrm{a}$ \\
\hline \multirow{2}{*}{ Tirosina } & Trat. 25g & $81,59 \mathrm{~b}$ & $67,76 \mathrm{~b}$ & $86,50 \mathrm{~b}$ & $76,60 \mathrm{~b}$ & $69,79 \mathrm{a}$ & $64,14 \mathrm{~b}$ & $73,35 \mathrm{~b}$ & $67,62 \mathrm{~b}$ \\
& Trat. 50g & $88,13 \mathrm{a}$ & $85,67 \mathrm{a}$ & $91,53 \mathrm{a}$ & $83,69 \mathrm{a}$ & $68,84 \mathrm{a}$ & $77,74 \mathrm{a}$ & $79,93 \mathrm{a}$ & $77,42 \mathrm{a}$ \\
\hline \multirow{2}{*}{ Sererina } & Trat. 25g & $85,59 \mathrm{~b}$ & $78,69 \mathrm{~b}$ & $90,00 \mathrm{~b}$ & $84,45 \mathrm{~b}$ & $83,49 \mathrm{a}$ & $71,10 \mathrm{a}$ & $79,28 \mathrm{~b}$ & $76,65 \mathrm{~b}$ \\
& Trat. 50g & $91,87 \mathrm{a}$ & $90.06 \mathrm{a}$ & $93,64 \mathrm{a}$ & $93,14 \mathrm{a}$ & $85,49 \mathrm{a}$ & $83,49 \mathrm{~b}$ & $85,36 \mathrm{a}$ & $85,28 \mathrm{a}$ \\
\hline \multirow{2}{*}{ Glutamina } & Trat. 25g & $88,95 \mathrm{~b}$ & $93,42 \mathrm{~b}$ & $94,00 \mathrm{a}$ & $94,62 \mathrm{~b}$ & $95,08 \mathrm{a}$ & $85,32 \mathrm{a}$ & $86,39 \mathrm{~b}$ & $88,47 \mathrm{~b}$ \\
& Trat. 50g & $94,16 \mathrm{a}$ & $97,10 \mathrm{a}$ & $95,23 \mathrm{a}$ & $98,55 \mathrm{a}$ & $96,04 \mathrm{a}$ & $89,78 \mathrm{a}$ & $91,24 \mathrm{a}$ & $92,63 \mathrm{a}$ \\
\hline \multirow{2}{*}{ Prolina } & Trat. 25g & $85,67 \mathrm{~b}$ & $87,31 \mathrm{~b}$ & $87,21 \mathrm{~b}$ & $91,47 \mathrm{~b}$ & $85,59 \mathrm{~b}$ & $74,36 \mathrm{~b}$ & $81,42 \mathrm{~b}$ & $88,33 \mathrm{a}$ \\
& Trat. 50g & $92,46 \mathrm{a}$ & $95,60 \mathrm{a}$ & $92,36 \mathrm{a}$ & $95,58 \mathrm{a}$ & $90,97 \mathrm{a}$ & $87,18 \mathrm{a}$ & $88,85 \mathrm{a}$ & $91,49 \mathrm{a}$ \\
\hline \multirow{2}{*}{ Alanina } & Trat. 25g & $80,33 \mathrm{~b}$ & $57,12 \mathrm{~b}$ & $89,81 \mathrm{a}$ & $68,21 \mathrm{~b}$ & $76,26 \mathrm{~b}$ & $65,07 \mathrm{~b}$ & $62,30 \mathrm{~b}$ & $49,28 \mathrm{a}$ \\
& Trat. 50g & $88,71 \mathrm{a}$ & $84,89 \mathrm{a}$ & $91,55 \mathrm{a}$ & $92,09 \mathrm{a}$ & $84,49 \mathrm{a}$ & $78,25 \mathrm{a}$ & $77,01 \mathrm{a}$ & $73,40 \mathrm{a}$ \\
\hline
\end{tabular}

Valores na coluna, para uma mesma variável, com letras distintas diferem entre si, pelo teste $\mathrm{F}(\mathrm{P}<0,05)$

1: RR = ração-referência, TIM = trigo integral moído, GT = germe de trigo, FTC = farinha de trigo clara, FTE = farinha de trigo escura, FTH = farelo de trigo para uso humano, FTA = farelo de trigo para uso animal, FTG = farelo de trigo grosso. 
TABELA 8 - Coeficiente de digestibilidade verdadeira (\%) da soma total dos aminoácidos (aa), da soma dos aa não essenciais, da soma dos aa essenciais e da soma dos aa limitantes dos alimentos ${ }^{1}$, segundo o nível de consumo.

\begin{tabular}{lccccccccc}
\hline \multicolumn{1}{c}{ Aminoácido } & Método & RR & TIM & GT & FTC & FTE & FTH & FTA & FTG \\
\hline \multirow{2}{*}{ Totais } & Trat. 25g & $85,10 \mathrm{~b}$ & $78,11 \mathrm{~b}$ & $89,35 \mathrm{~b}$ & $80,70 \mathrm{~b}$ & $79,53 \mathrm{~b}$ & $69,87 \mathrm{~b}$ & $75,12 \mathrm{~b}$ & $75,11 \mathrm{~b}$ \\
& Trat. 50g & $91,83 \mathrm{a}$ & $93,03 \mathrm{a}$ & $93,43 \mathrm{a}$ & $95,60 \mathrm{a}$ & $79,53 \mathrm{a}$ & $84,58 \mathrm{a}$ & $86,16 \mathrm{a}$ & $86,25 \mathrm{a}$ \\
\hline \multirow{2}{*}{$\mathrm{AA}_{\mathrm{NE}}{ }^{2}$} & Trat. 25g & $84,92 \mathrm{~b}$ & $77,53 \mathrm{~b}$ & $89.97 \mathrm{~b}$ & $82,84 \mathrm{~b}$ & $83,27 \mathrm{~b}$ & $71,39 \mathrm{~b}$ & $76,84 \mathrm{~b}$ & $73,36 \mathrm{~b}$ \\
& Trat. 50g & $92,87 \mathrm{a}$ & $95,02 \mathrm{a}$ & $94,22 \mathrm{a}$ & $96,49 \mathrm{a}$ & $91,94 \mathrm{a}$ & $86,38 \mathrm{a}$ & $88,30 \mathrm{a}$ & $89,27 \mathrm{a}$ \\
\hline \multirow{2}{*}{$\mathrm{AA}_{\mathrm{E}}{ }^{2}$} & Trat. 25g & $85,24 \mathrm{~b}$ & $78,55 \mathrm{~b}$ & $88,89 \mathrm{~b}$ & $79,10 \mathrm{~b}$ & $76,73 \mathrm{~b}$ & $68,74 \mathrm{~b}$ & $73,83 \mathrm{~b}$ & $74,92 \mathrm{~b}$ \\
& Trat. 50g & $90,65 \mathrm{a}$ & $88,91 \mathrm{a}$ & $92,57 \mathrm{a}$ & $93,79 \mathrm{a}$ & $84,93 \mathrm{a}$ & $82,53 \mathrm{a}$ & $83,32 \mathrm{a}$ & $81,69 \mathrm{a}$ \\
\hline \multirow{2}{*}{$\mathrm{LIM}^{2,3}$} & Trat. 25g & $90,85 \mathrm{a}$ & $80,70 \mathrm{~b}$ & $92,19 \mathrm{a}$ & $73,14 \mathrm{~b}$ & $85,09 \mathrm{a}$ & $74,86 \mathrm{~b}$ & $80,74 \mathrm{~b}$ & $81,28 \mathrm{~b}$ \\
& Trat. 50g & $93,58 \mathrm{a}$ & $91,08 \mathrm{a}$ & $94,89 \mathrm{a}$ & $97,90 \mathrm{a}$ & $85,09 \mathrm{a}$ & $86,33 \mathrm{a}$ & $87,49 \mathrm{a}$ & $85,66 \mathrm{a}$ \\
\hline
\end{tabular}

Valores na coluna, para uma mesma variável, com letras distintas diferem entre si, pelo teste $\mathrm{F}(\mathrm{P}<0,05)$

1: RR = ração-referência, TIM = trigo integral moído, GT = germe de trigo, FTC = farinha de trigo clara, FTE = farinha de trigo escura, FTH = farelo de trigo para uso humano, FTA = farelo de trigo para uso animal, FTG = farelo de trigo grosso.

2: AANE = aminoácidos não essenciais, AAE = aminoácidos essenciais e LIM = aminoácidos limitantes

3: Metionina, treonina e lisina.

Este efeito pode ser devido ao maior catabolismo protéico das aves que receberam menor quantidade de alimentos, embora não seja possível comprovar neste experimento sua real extensão. O nível de 50 gramas de ingestão apresentou CDVaa mais altos em todos os tratamentos quando comparados ao nível de 25 gramas $(\mathrm{P}<0,05)$, com exceção aos aminoácidos limitantes da ração-referência, do germe de trigo e da farinha de trigo escura, que foram significativamente semelhantes $(\mathrm{P}>0,05)$ entre os dois tratamentos.

Neste ponto é importante estabelecer uma relação entre os valores obtidos para a digestibilidade de aminoácidos e os valores encontrados na EMV e EMVn desses mesmos tratamentos (BORGES et al., 1990). Nos resultados de EMV e EMVn, obtidos pelos citados autores, observou-se que a EMV apresentou diferenças significativas entre os vários alimentos, o que não acorreu com a EMVn, que apresentou valores estatisticamente iguais para todos os alimentos, com exceção a FTC. Ora, se no trabalho de Borges et al. (1990) a correção do excretado endógeno foi feita para as duas medidas, torna-se evidente que uma porção extra e não quantificável de nitrogênio, possivelmente sob a forma de aminoácidos endógenos, poderia estar interferindo sobre os valores de EMV, o que se torna evidente depois de realizadas as devidas correções, pois essas diferenças estatísticas desaparecem na EMVn.

Esse fato pode ser confirmado observando-se a CDVaa dos dois níveis de ingestão, no presente trabalho. Estes resultados estão de acordo com Pesti (1984), que afirma que o baixo nível de ingestão pode levar a uma mobilização de tecidos que modifica a quantidade de nitrogênio excretada.

\section{CONCLUSÕES}

Nas condições em que se realizou este trabalho pode-se concluir que:

Os coeficientes de digestibilidade aparente dos aminoácidos são afetados pela quantidade de alimento ingerido, e nos níveis de consumo menores os valores de CDAaa são significativamente menores. Do mesmo modo, os coeficientes de digestibilidade verdadeira do total de aminoácidos, da soma dos aminoácidos essenciais, da soma dos aminoácidos não essenciais e da soma dos aminoácidos limitantes para a maioria dos alimentos apresentaram diferenças significativas entre os níveis de consumo. Neste caso recomenda-se que, para este tipo de avaliação, sejam utilizados consumos iguais ou maiores que 50 gramas de alimento. 


\section{REFERÊNCIAS BIBLIOGRÁFICAS}

ALBINO, L. T. F. Sistemas de avaliação nutricional de alimentos e suas aplicações na formulação de rações para frangos de corte. 1991. 55 f. Tese (Doutorado em Zootecnia) - Universidade Federal de Viçosa, Viçosa, 1991.

BORGES, F. M. O. et al. Metodologia de alimentação forçada em aves: I efeito dos níveis de consumo de alimento na avaliação da energia metabolizável. In: REUNIÃO ANUAL DA SOCIEDADE BRASILEIRA DE ZOOTECNIA, 35., 1990, Botucatu. Anais... Botucatu: SBZ, $1990 . \quad$ Disponível em: <http://www.sbz.org.br/scripts/anais1998>. Acesso em: 20 out. 2003.

DALE, N.; FULLER, H. L. Correlation of protein content of feedstuffs with the magnitude of nitrogen correction in true metabolizable energy determinations. Poultry Science, Champaign, v. 63, p. 1008-1012, 1984.

MEJÍA, A. M. G.; FERREIRA, W. M. Métodos de avaliação da disponibilidade da proteína e dos aminoácidos nos alimentos para não ruminantes. In: SIMPÓSIO INTERNACIONAL DE PRODUÇÃO DE MONOGÁSTRICOS, 1., 1996, Seropédica, RJ. Anais... Seropédica: [s.n.], 1996.

MOTTER, J. H. Fundamental principies of HPLC. [S.l.]: Shimadzu, 1991.49 p.
PESTI, G. M. Influence of substitution method and of food intake on bioassays to determine metabolisable energy with chickens. British Poultry Science, London, v. 25, p. 495-504, 1984.

PESTI, G. M.; DALE, N. M.; FARRELL, D. J. A comparison of methods to determine the metabolizable energy of feather meal. Poultry Science, Champaign, v. 68, p. 443-446, 1988.

PUPA, J. M. R. et al. Cecectomia em galos sob anestesia local e incisão abdominal. Arquivo Brasileiro de Medicina Veterinária e Zootecnia, Belo Horizonte, v. 50, p. 531-535, 1998.

ROSTAGNO, H. S. et al. Composição de alimentos e exigências nutricionais de aves e suínos. 19. ed. Viçosa: UFV, 1985. 59 p.

SIBBALD, I. R. A bioassay for true metabolizable energy in feedingstuffs. Poultry Science, Champaign, v. 55, p. 303-308, 1976.

SIBBALD, I. R. The effect of level of feed input on true metabolizable energy values. Poultry Science, Champaign, v. 56, p. 1662-1663, 1977.

SIBBALD, I. R. The effects dietary cellulose and ssand on the combined metabolic plus endogenous energy and amino acid output adult cockerels. Poultry Science, Champaign, v. 59, p. 836-844, 1980. 